\title{
COMPLEX OF INJECTIVE WORDS REVISITED
}

\author{
WEE LIANG GAN
}

\begin{abstract}
We give a simple proof that (a generalization of) the complex of injective words has vanishing homology in all except the top degree.
\end{abstract}

\section{INTRODUCTION}

Let $A$ be a finite set. An injective word of length $r \geqslant 0$ is a sequence $\left(a_{1}, \ldots, a_{r}\right)$ of pairwise distinct elements of $A$. Let $K(A)$ be the semi-simplicial set whose $(r-1)$-simplices are the injective words of length $r$, for every $r \geqslant 1$. The face maps of $K(A)$ are defined by

$$
d_{i-1}\left(a_{1}, \ldots, a_{r}\right):=\left(a_{1}, \ldots, \widehat{a_{i}}, \ldots, a_{r}\right) \quad \text { for } i=1, \ldots, r,
$$

where $\widehat{x}$ means that the entry $x$ is omitted. In other words, $K(A)$ is the semi-simplicial set of ordered simplices of the abstract simplex whose set of vertices is $A$. We write $|K(A)|$ for the geometric realization of $K(A)$. For example, if $A=\{a, b\}$, then $|K(A)|$ is homeomorphic to a circle:

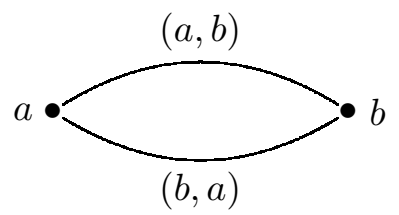

In a 1979 paper, Farmer [4, Theorem 5] shows that the reduced homology of $K(A)$ vanishes in all degrees $\neq|A|-1$. Subsequently, a new proof was found by Björner and Wachs [2, Theorem 6.1] using their theory of CL-shellable posets. A simpler proof of Farmer's result was given by Kerz [6. Theorem 1] in 2004. Both the proofs of Farmer and Kerz proceed by somewhat ad hoc calculations. More recently, topological proofs of Farmer's result are given by Bestvina [1, Claim in the proof of Proposition 6] and Randal-Williams [9, Proposition 3.2]. We should mention that Björner-Wachs and Randal-Williams actually proved the stronger result that $|K(A)|$ is homotopy equivalent to a wedge of spheres of dimension $|A|-1$.

The purpose of our present note is to give a simple and natural algebraic proof of Farmer's result; indeed, our proof is a straightforward exercise on the spectral sequence of a filtered complex. Our interest in this result stems from the crucial role it plays in Quillen's method [8] for proving homological stability of the symmetric groups. Following Hatcher and Wahl [5], we shall formulate and prove a slightly more general theorem so that it can be applied in the proof of homological stability of wreath-product groups.

2010 Mathematics Subject Classification. $18 \mathrm{G} 35$.

Key words and phrases. injective word, homological stability, wreath-product group. 
Notation 1. We write $S_{n}$ for the symmetric group on $\{1, \ldots, n\}$. For any group $G$, we write $G_{n}$ for the wreath-product group $G \prec S_{n}$, that is, $G_{n}:=S_{n} \ltimes G^{n}$. In particular, $G_{0}$ is the trivial group.

\section{THE MAIN RESULT}

Recall that $A$ denotes a finite set. Let $\Gamma$ be a nonempty set. We define a chain complex $C_{*}(A)$ concentrated in degrees $0, \ldots,|A|$ as follows. Let $C_{r}(A)$ be the free abelian group generated by the set $\Delta_{r}(A)$ consisting of all elements of the form $\left(a_{1}, \ldots, a_{r}, \gamma_{1}, \ldots, \gamma_{r}\right)$ where $a_{1}, \ldots, a_{r}$ are pairwise distinct elements of $A$, and $\gamma_{1}, \ldots, \gamma_{r}$ are any elements of $\Gamma$; in particular, $C_{r}(A)=\mathbb{Z}$ if $r=0$. The differential is defined by

$$
d\left(a_{1}, \ldots, a_{r}, \gamma_{1}, \ldots, \gamma_{r}\right):=\sum_{i=1}^{r}(-1)^{i-1}\left(a_{1}, \ldots, \widehat{a_{i}}, \ldots, a_{r}, \gamma_{1}, \ldots, \widehat{\gamma_{i}}, \ldots, \gamma_{r}\right) .
$$

In particular, $d\left(a_{1}, \gamma_{1}\right)=1$.

For any chain complex $C_{*}$ and positive integer $p$, we shall write $C_{*-p}$ for the $p$-fold suspension of $C_{*}$.

Remark 2. If $\Gamma$ is a singleton set, then $C_{*}(A)$ is the augmented chain complex of $K(A)$ with degrees shifted up by 1 . (Topologically, it is more natural to place a word of length $r$ in degree $r-1$. Algebraically, it seems more natural for us to place a word of length $r$ in degree $r$.)

If $\Gamma$ is a group $G$, then $C_{*}(A)$ is the augmented chain complex of the semi-simplicial set $W_{|A|}(\emptyset,\{1\})$ (defined by Randal-Williams and Wahl in [10, Definition 2.1]) with degrees shifted up by 1, associated to the category $\mathrm{FI}_{G}$ (defined by Sam and Snowden in [12]).

Theorem 3. If $r<|A|$, then $H_{r}\left(C_{*}(A)\right)=0$.

Proof. Set $n=|A|$. We use induction on $n$. The base case $n=0$ is trivial.

Suppose $n>0$. Choose and fix an element $a \in A$. For each $r \geqslant 0$, there is an increasing filtration on $C_{r}(A)$ defined by letting $F_{p} C_{r}(A)$ be the subgroup spanned by all elements $\left(a_{1}, \ldots, a_{r}, \gamma_{1}, \ldots, \gamma_{r}\right)$ such that none of $a_{p+1}, \ldots, a_{r}$ is equal to $a$. This gives an increasing filtration on the complex $C_{*}(A)$ and hence a first-quadrant spectral sequence:

$$
E_{p, q}^{1}=H_{p+q}\left(F_{p} C_{*}(A) / F_{p-1} C_{*}(A)\right) \Rightarrow H_{p+q}\left(C_{*}(A)\right) .
$$

Observe that $F_{0} C_{*}(A)=C_{*}(A \backslash\{a\})$. For each $p \geqslant 1$, there is an isomorphism of chain complexes:

$$
F_{p} C_{*}(A) / F_{p-1} C_{*}(A) \cong \bigoplus_{\left(a_{1}, \ldots, a_{p-1}, a, \gamma_{1}, \ldots, \gamma_{p}\right) \in \Delta_{p}(A)} C_{*-p}\left(A \backslash\left\{a_{1}, \ldots, a_{p-1}, a\right\}\right)
$$

where an element on the left hand side represented by $\left(a_{1}, \ldots, a_{r}, \gamma_{1}, \ldots, \gamma_{r}\right) \in F_{p} C_{r}(A)$ with $a_{p}=a$ is identified, on the right hand side, with the element $\left(a_{p+1}, \ldots, a_{r}, \gamma_{p+1}, \ldots, \gamma_{r}\right)$ in the direct summand $C_{r-p}\left(A \backslash\left\{a_{1}, \ldots, a_{p-1}, a\right\}\right)$ indexed by $\left(a_{1}, \ldots, a_{p}, \gamma_{1}, \ldots, \gamma_{p}\right) \in \Delta_{p}(A)$.

By the induction hypothesis, one has:

$$
\begin{gathered}
E_{0, q}^{1}=0 \quad \text { whenever } \quad q<n-1 ; \\
E_{p, q}^{1}=0 \quad \text { whenever } \quad p \geqslant 1 \text { and } p+q<n .
\end{gathered}
$$


Therefore, it only remains to show that $E_{0, n-1}^{\infty}=0$.

We have:

$$
E_{0, n-1}^{1}=H_{n-1}\left(C_{*}(A \backslash\{a\})\right), \quad E_{1, n-1}^{1}=\bigoplus_{\gamma_{1} \in \Gamma} H_{n-1}\left(C_{*}(A \backslash\{a\})\right) .
$$

The restriction of the differential $d^{1}: E_{1, n-1}^{1} \rightarrow E_{0, n-1}^{1}$ to each direct summand in the above decomposition of $E_{1, n-1}^{1}$ is the identity map on $H_{n-1}\left(C_{*}(A \backslash\{a\})\right)$; this follows from the identity

$$
\begin{aligned}
d\left(a, a_{2}, \ldots, a_{n}, \gamma_{1}, \ldots, \gamma_{n}\right)=\left(a_{2}, \ldots, a_{n}, \gamma_{2}, \ldots, \gamma_{n}\right) & \\
& -\sum_{i=1}^{n-1}(-1)^{i-1}\left(a, a_{2}, \ldots, \widehat{a_{i+1}}, \ldots, \gamma_{1}, \gamma_{2}, \ldots, \widehat{\gamma_{i+1}}, \ldots\right) .
\end{aligned}
$$

In particular, the map $d^{1}: E_{1, n-1}^{1} \rightarrow E_{0, n-1}^{1}$ is surjective. It follows that $E_{0, n-1}^{2}=0$, and we are done.

Remark 4. It was pointed out to us by the referee that the last step in the above proof can be replaced by the following argument. Fixing an element $e \in \Gamma$, the map

$$
\left(a_{1}, \ldots, a_{r}, \gamma_{1}, \ldots, \gamma_{r}\right) \mapsto\left(a, a_{1}, \ldots, a_{r}, e, \gamma_{1}, \ldots, \gamma_{r}\right)
$$

gives a null-homotopy for the inclusion map $C_{*}(A-\{a\}) \rightarrow C_{*}(A)$. Thus, the edge map $E_{0, n-1}^{1} \longrightarrow E_{0, n-1}^{\infty} \subset H_{n-1}\left(C_{*}(A)\right)$ is zero; hence, $E_{0, n-1}^{\infty}=0$.

Remark 5. Let $n=|A|$. Since $H_{n}\left(C_{*}(A)\right)$ is a subgroup of the free abelian group $C_{n}(A)$, it is a free abelian group; we make the following observations on its rank.

(i) Suppose $|\Gamma|=\infty$. If $n=|A| \geqslant 1$, then $H_{n}\left(C_{*}(A)\right)$ is a free abelian group of infinite rank. This is clear if $n=1$. For $n>1$, it follows from noticing that by induction, the kernel of $d^{1}: E_{1, n-1}^{1} \rightarrow E_{0, n-1}^{1}$ (in the spectral sequence in the proof above) is a free abelian group of infinite rank, and which is $E_{1, n-1}^{\infty}$.

(ii) Suppose $|\Gamma|=\ell<\infty$. If $n=|A| \geqslant 1$, then it follows from Theorem 3 and the Euler-Poincaré principle that $H_{n}\left(C_{*}(A)\right)$ is a free abelian group of rank $d(\ell)_{n}$, where

$$
d(\ell)_{n}:=\sum_{i=0}^{n} \frac{(-1)^{i} n ! \ell^{n-i}}{i !} .
$$

For $\ell=1$, this observation is due to Farmer [4, Remark on page 613] and Reiner-Webb [11, Proposition 2.1]. It is well known that $d(1)_{n}$ is equal to the number of derangements in the symmetric group $S_{n}$. Let us give a similar interpretation of $d(\ell)_{n}$ for any $\ell \geqslant 1$. Fixing an element $e \in \Gamma$, we claim that $d(\ell)_{n}$ is the number of elements $\left(\pi, \gamma_{1}, \ldots, \gamma_{n}\right) \in S_{n} \times \Gamma^{n}$ such that: if $1 \leqslant a \leqslant n$ and $\pi(a)=a$, then $\gamma_{a} \neq e$. To see this, let

$$
T_{a}:=\left\{\left(\pi, \gamma_{1}, \ldots, \gamma_{n}\right) \in S_{n} \times \Gamma^{n} \mid \pi(a)=a \text { and } \gamma_{a}=e\right\} \quad \text { for } a=1, \ldots, n .
$$

Then, for any $1 \leqslant a_{1}<\cdots<a_{i} \leqslant n$, one has $\left|T_{a_{1}} \cap \cdots \cap T_{a_{i}}\right|=(n-i) ! \ell^{n-i}$. Hence, by the inclusion-exlusion principle, we have $d(\ell)_{n}=\left|S_{n} \times \Gamma^{n}\right|-\left|T_{1} \cup \cdots \cup T_{n}\right|$, as claimed.

We note that for any group $G$, the wreath-product group $G_{n}$ (see Notation 11) acts on $\{1, \ldots, n\} \times G$ by $\left(\pi ; g_{1}, \ldots g_{n}\right) \cdot(a, \gamma):=\left(\pi(a), g_{a} \gamma\right)$, where $\left(\pi ; g_{1}, \ldots, g_{n}\right) \in G_{n}$ and $(a, \gamma) \in$ 
$\{1, \ldots, n\} \times G$. When $G$ is a finite group of order $\ell$, the integer $d(\ell)_{n}$ is equal to the number of elements of $G_{n}$ which has no fixed point in $\{1, \ldots, n\} \times G$.

\section{Application to homological stability}

Nakaoka [7, Corollary 6.7] proved that the natural inclusion map $S_{n-1} \rightarrow S_{n}$ induces an isomorphism in homology $H_{m}\left(S_{n-1}\right) \rightarrow H_{m}\left(S_{n}\right)$ if $n>2 m$. His result was generalized by Hatcher and Wahl [5, Proposition 1.6] to wreath-product groups (although as they noted in their paper the generalization might have been known for a long time).

Corollary 6. Let $G$ be a group. The natural inclusion map $G \backslash S_{n-1} \rightarrow G\left(S_{n}\right.$ induces an isomorphism in homology $H_{m}\left(G \backslash S_{n-1}\right) \rightarrow H_{m}\left(G \prec S_{n}\right)$ if $n>2 m$.

Corollary 6 follows from Theorem 3 by a standard argument of Quillen; see [5, Section 5]. We give the details of this argument below since our injectivity range $n>2 m$ is better than the one stated in [5, Proposition 1.6] by 1.

From now on, we set $A=\{1, \ldots, n\}$ and $\Gamma=G$, where $n$ is an integer $\geqslant 1$ and $G$ is a group.

There is a natural action of $G_{n}$ (see Notation 1) on $C_{r}(A)$ defined by

$$
\left(\pi ; g_{1}, \ldots, g_{n}\right) \cdot\left(a_{1}, \ldots, a_{r}, \gamma_{1}, \ldots, \gamma_{r}\right):=\left(\pi\left(a_{1}\right), \ldots, \pi\left(a_{r}\right), g_{a_{1}} \gamma_{1}, \ldots, g_{a_{r}} \gamma_{r}\right),
$$

where $\left(\pi ; g_{1}, \ldots, g_{n}\right) \in G_{n}$ and $\left(a_{1}, \ldots, a_{r}, \gamma_{1}, \ldots, \gamma_{r}\right) \in C_{r}(A)$. Define the map

$$
d_{i-1}: C_{r}(A) \longrightarrow C_{r-1}(A) \quad \text { for } i=1, \ldots, r,
$$

by

$$
d_{i-1}\left(a_{1}, \ldots, a_{r}, \gamma_{1}, \ldots, \gamma_{r}\right):=\left(a_{1}, \ldots, \widehat{a_{i}}, \ldots, a_{r}, \gamma_{1}, \ldots, \widehat{\gamma_{i}}, \ldots, \gamma_{r}\right) .
$$

Since the map $d_{i-1}$ is $G_{n}$-equivariant, there is an induced map

$$
\left(d_{i-1}\right)_{*}: H_{*}\left(G_{n} ; C_{r}(A)\right) \rightarrow H_{*}\left(G_{n} ; C_{r-1}(A)\right) .
$$

The group $G_{n}$ acts transitively on the basis $\Delta_{r}(A)$ of $C_{r}(A)$. For any $x \in \Delta_{r}(A)$, we write $\operatorname{Stab}(x)$ for its stablizer in $G_{n}$. By Shapiro's lemma, the natural inclusion map $\operatorname{Stab}(x) \rightarrow G_{n}$ and the map $\mathbb{Z} \rightarrow C_{r}(A), \lambda \mapsto \lambda x$ induce an isomorphism

$$
\alpha(x)_{*}: H_{*}(\operatorname{Stab}(x)) \longrightarrow H_{*}\left(G_{n} ; C_{r}(A)\right) .
$$

Denote by $e \in G$ the identity element. Let

$$
x_{r}:=(n-r+1, \ldots, n, e, \ldots, e) \in C_{r}(A) .
$$

Then $\operatorname{Stab}\left(x_{r}\right)=G_{n-r} \leqslant G_{n}$. In particular, $x_{0}=1 \in \mathbb{Z}$ and $\operatorname{Stab}\left(x_{0}\right)=G_{n}$. We write $\iota: \operatorname{Stab}\left(x_{r}\right) \rightarrow \operatorname{Stab}\left(x_{r-1}\right)$ for the natural inclusion map.

Lemma 7. For every $i=1, \ldots, r$, the following diagram commutes:

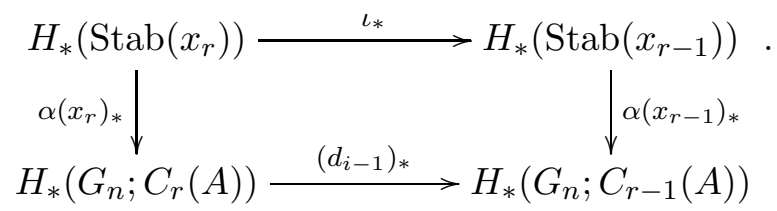


Proof. The diagram clearly commutes for $i=1$ because $d_{0}\left(x_{r}\right)=x_{r-1}$.

Suppose $i>1$. Let $y:=d_{i-1}\left(x_{r}\right)$, so

$$
y=(n-r+1, \ldots, n \widehat{-r+} i, \ldots, n, e, \ldots, e) \in C_{r-1}(A) .
$$

Write $\jmath: \operatorname{Stab}\left(x_{r}\right) \rightarrow \operatorname{Stab}(y)$ for the natural inclusion map. Then there is a commuting diagram:

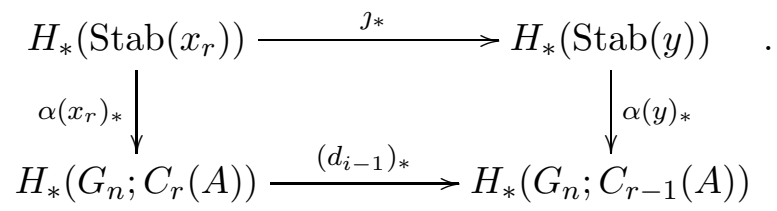

Let $\mu$ be the cyclic permutation $(n-r+1, \ldots, n-r+i) \in S_{n}$ and let

$$
t:=(\mu ; e, \ldots, e) \in G_{n} .
$$

Then $t$ has the properties that $t \cdot y=x_{r-1}$ and

$$
t u t^{-1}=u \quad \text { for each } u \in \operatorname{Stab}\left(x_{r}\right) .
$$

We have a commuting diagram

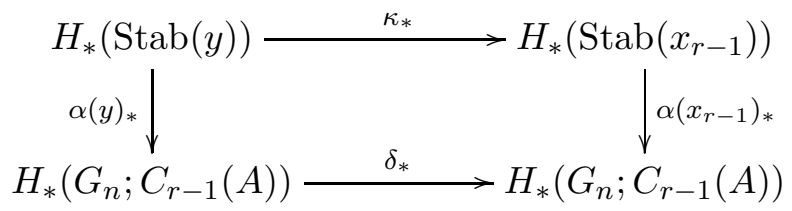

where the top arrow $\kappa_{*}$ is induced by the homomorphism $\kappa: \operatorname{Stab}(y) \rightarrow \operatorname{Stab}\left(x_{r-1}\right), u \mapsto$ $t u t^{-1}$ and the bottom arrow $\delta_{*}$ is induced by the inner automorphism $G_{n} \rightarrow G_{n}, u \mapsto t u t^{-1}$ and the map $C_{r-1}(A) \rightarrow C_{r-1}(A), x \mapsto t \cdot x$.

By (2), we have $\kappa \circ \jmath=\iota$, so $\kappa_{*} \circ \jmath_{*}=\iota_{*}$. By [3, Proposition III.8.1], the homomorphism $\delta_{*}$ is the identity map on $H_{*}\left(G_{n} ; C_{r-1}(A)\right)$. Therefore, it follows from our two commuting diagrams (11) and (3) that

$$
\begin{aligned}
\alpha\left(x_{r-1}\right)_{*} \circ \iota_{*}=\alpha\left(x_{r-1}\right)_{*} \circ \kappa_{*} \circ \jmath_{*}=\delta_{*} \circ \alpha(y)_{*} \circ \jmath^{*} & \\
& =\delta_{*} \circ\left(d_{i-1}\right)_{*} \circ \alpha\left(x_{r}\right)_{*}=\left(d_{i-1}\right)_{*} \circ \alpha\left(x_{r}\right)_{*} .
\end{aligned}
$$

We are now ready to prove Corollary 6 .

Proof of Corollary [6. We use induction on $m$. The base case $m=0$ is trivial.

Suppose $m \geqslant 1$. Let $n>2 m$.

Choose any free resolution $\cdots \rightarrow F_{1} \rightarrow F_{0} \rightarrow \mathbb{Z}$ of $\mathbb{Z}$ over $\mathbb{Z} G_{n}$. By taking the tensor product over $G_{n}$ of the two chain complexes $C_{*}(A)$ and $F_{*}$, we obtain a first-quadrant double complex $D$ with $D_{r, s}:=F_{s} \otimes_{G_{n}} C_{r}(A)$. Let $\operatorname{Tot}_{*}(D)$ be the total complex of $D$. From Theorem 3 and the spectral sequence associated to the horizontal filtration of $\operatorname{Tot}_{*}(D)$, we deduce that $H_{i}\left(\operatorname{Tot}_{*}(D)\right)=0$ for each $i \leqslant n-1$.

We now consider the spectral sequence associated to the vertical filtration of $\operatorname{Tot}_{*}(D)$. Since $H_{i}\left(\operatorname{Tot}_{*}(D)\right)=0$ for each $i \leqslant n-1$, this spectral sequence has:

$$
E_{r, s}^{\infty}=0 \quad \text { if } \quad r+s \leqslant n-1 .
$$


The $E^{1}$-terms of the spectral sequence are:

$$
E_{r, s}^{1}=H_{s}\left(F_{*} \otimes_{G_{n}} C_{r}(A)\right)=H_{s}\left(G_{n} ; C_{r}(A)\right) .
$$

The differential $d^{1}: E_{r, s}^{1} \rightarrow E_{r-1, s}^{1}$ is the map

$$
d_{*}=\sum_{i=1}^{r}(-1)^{i-1}\left(d_{i-1}\right)_{*}: H_{s}\left(G_{n} ; C_{r}(A)\right) \longrightarrow H_{s}\left(G_{n} ; C_{r-1}(A)\right) .
$$

Recall that $G_{n-r}=\operatorname{Stab}\left(x_{r}\right)$. We shall identify $E_{r, s}^{1}$ with $H_{s}\left(G_{n-r}\right)$ via the isomorphism $\alpha\left(x_{r}\right)_{*}: H_{s}\left(G_{n-r}\right) \rightarrow H_{s}\left(G_{n} ; C_{r}(A)\right)$. Under this identification, we see from Lemma 7 that the differential $d^{1}: E_{r, s}^{1} \rightarrow E_{r-1, s}^{1}$ is:

- the map $\iota_{*}: H_{s}\left(G_{n-r}\right) \rightarrow H_{s}\left(G_{n-r+1}\right)$ if $r$ is odd;

- the zero map if $r$ is even.

Therefore, row $s$ on the $E^{1}$-page of the spectral sequence is:

$$
H_{s}\left(G_{n}\right) \stackrel{\iota_{*}}{\longleftarrow} H_{s}\left(G_{n-1}\right) \stackrel{0}{\longleftarrow} H_{s}\left(G_{n-2}\right) \stackrel{\iota_{*}}{\longleftarrow} H_{s}\left(G_{n-3}\right) \stackrel{0}{\longleftarrow} H_{s}\left(G_{n-4}\right) \stackrel{\iota_{*}}{\longleftarrow} \cdots,
$$

where the leftmost term is in column 0 .

Our goal is to show that $\iota_{*}: H_{m}\left(G_{n-1}\right) \rightarrow H_{m}\left(G_{n}\right)$ is an isomorphism, or equivalently, that the differential $d^{1}: E_{1, m}^{1} \rightarrow E_{0, m}^{1}$ is an isomorphism. Note that $E_{1, m}^{2}$ is the kernel of $d^{1}: E_{1, m}^{1} \rightarrow E_{0, m}^{1}$, and $E_{0, m}^{2}$ is the cokernel of $d^{1}: E_{1, m}^{1} \rightarrow E_{0, m}^{1}$. Therefore, we have to show that $E_{1, m}^{2}=0$ and $E_{0, m}^{2}=0$. We shall use the following:

Claim. If $r+2 s \leqslant 2 m+1$ and $s<m$, then $E_{r, s}^{2}=0$.

Proof of Claim. We have:

$$
2 s \leqslant 2 m+1-r<n-r+1 .
$$

When $r$ is even, we have the stronger inequality:

$$
2 s \leqslant 2 m-r<n-r .
$$

Hence, it follows by the induction hypothesis that in row $s$ of the $E^{1}$-page of the spectral sequence, we have:

$$
\cdots \longleftarrow H_{s}\left(G_{n-r+1}\right) \stackrel{d_{r, s}^{1}}{\longleftarrow} H_{s}\left(G_{n-r}\right) \stackrel{d_{r+1, s}^{1}}{\longleftarrow} H_{s}\left(G_{n-r-1}\right) \longleftarrow \cdots
$$

where

- $d_{r, s}^{1}$ is an isomorphism and $d_{r+1, s}^{1}$ is the zero map if $r$ is odd;

- $d_{r . s}^{1}$ is the zero map and $d_{r+1, s}^{1}$ is an isomorphism if $r$ is even. Hence, $E_{r, s}^{2}=0$.

We have proven the Claim.

The above Claim implies that $E_{1, m}^{2}=E_{1, m}^{\infty}$ and $E_{0, m}^{2}=E_{0, m}^{\infty}$. Indeed, for $k \geqslant 2$, a differential on the $E^{k}$-page has target $E_{1, m}^{k}$ or $E_{0, m}^{k}$ only if it starts at $E_{k+1, m-k+1}^{k}$ or $E_{k, m-k+1}^{k}$ respectively, but the Claim implies that $E_{k+1, m-k+1}^{k}$ and $E_{k, m-k+1}^{k}$ are both zero.

Finally, since $n \geqslant 2 m+1 \geqslant 3$ and so

$$
m+1 \leqslant \frac{n+1}{2} \leqslant n-1,
$$


it follows from (44) that $E_{1, m}^{\infty}=0$ and $E_{0, m}^{\infty}=0$, so $E_{1, m}^{2}=0$ and $E_{0, m}^{2}=0$.

Remark 8. As the last step in the proof above shows, we only need to use the vanishing of $H_{r}\left(C_{*}(A)\right)$ for $|A| \geqslant 3$ and $r \leqslant \frac{|A|+1}{2}$.

Acknowledgments. I am grateful to Nathalie Wahl and the referee for their many helpful suggestions to improve the paper.

\section{REFERENCES}

[1] M. Bestvina. Homological stability of $\operatorname{Aut}\left(F_{n}\right)$ revisited. arXiv:1508.03876v1.

[2] A. Björner, M. Wachs. On lexicographically shellable posets. Trans. Amer. Math. Soc. 277 (1983), no. 1, 323-341.

[3] K.S. Brown. Cohomology of groups. Graduate Texts in Mathematics, 87. Springer-Verlag, New YorkBerlin, 1982.

[4] F.D. Farmer. Cellular homology for posets. Math. Japon. 23 (1978/79), no. 6, 607-613.

[5] A. Hatcher, N. Wahl. Stabilization for mapping class groups of 3-manifolds. Duke Math. J. 155 (2010), no. 2, 205-269. arXiv:0709.2173v4.

[6] M.C. Kerz. The complex of words and Nakaoka stability. Homology Homotopy Appl. 7 (2005), no. 1, 77-85. arXiv:math/0410261 v2.

[7] M. Nakaoka. Decomposition theorem for homology groups of symmetric groups. Ann. of Math. (2) 71 (1960), 16-42.

[8] D. Quillen. Notebooks, August 9, 1970.http://www.claymath.org/library/Quillen/Working_papers/quillen\%201971/1971-

[9] O. Randal-Williams. Homological stability for unordered configuration spaces. Q. J. Math. 64 (2013), no. 1, 303-326. arXiv:1105.5257v1.

[10] O. Randal-Williams, N. Wahl. Homological stability for automorphism groups. arXiv:1409.3541v3.

[11] V. Reiner, P. Webb. The combinatorics of the bar resolution in group cohomology. J. Pure Appl. Algebra 190 (2004), no. 1-3, 291-327.

[12] S. Sam, A. Snowden. Representations of categories of $G$-maps. arXiv:1410.6054v3.

Department of Mathematics, University of California, Riverside, CA 92521, USA.

E-mail address: wlgan@math.ucr.edu 